\title{
Estudio cualitativo sobre la autopercepción del estigma social en personas con esquizofrenia.
}

Qualitative study about the self perception of social stigma in people with schizophrenia.

\author{
Susana Ochoa a ${ }^{\text {, Francisco Martínez b }}{ }^{\text {, }}$ Maria Ribas c, Mar García-Franco d, Elisabeth López e, \\ Raul Villellas ${ }^{\mathrm{f}}$, Otilia Arenas ${ }^{\mathrm{g}}$, Irene Álvarez ${ }^{\mathrm{h}}$, Christian Cunyat ${ }^{\mathrm{i}}$, Sonia Vilamala ${ }^{\mathrm{j}}$, \\ Jaume Autonell ${ }^{\mathrm{k}}$, Esther Lobo ${ }^{1}$, Josep Maria Haro ${ }^{\mathrm{m}}$,
}

${ }^{a}$ Psicóloga. Coordinadora de la Unidad de Investigación y Desarrollo de Parc Sanitari Sant Joan de Déu. CIBERSAM, Sant Boi Llobregat (Barcelona). ${ }^{b}$ Trabajador social. Servicio de Rehabilitación Psicosocial El Prat de Llobregat Parc Sanitari Sant Joan de Déu (Barcelona). 'P ${ }^{c}$ sicóloga. Coordinadora de la Unidad de Terapia y Justicia Juvenil Til.lers Parc Sanitari Sant Joan de Déu. Mollet del Vallés (Barcelona). ${ }^{d} P$ sicológa. Servicio de Rehabilitación psicosocial Viladecans Parc Sanitari Sant Joan de Déu (Barcelona). ${ }^{e}$ Terapeuta ocupacional. Plan de Servicios Individualizados Parc Sanitari Sant Joan de Déu. Cerdanyola del Vallés (Barcelona). ${ }^{f}$ Psicopedagogo. Centro de Inserción Laboral de Esplugues de Llobregat Parc Sanitari Sant Joan de Déu (Barcelona). ${ }^{8}$ Trabajadora Social. Coordinadora de Servicios de Rehabilitación del Baix Llobregat Parc Sanitari Sant Joan de Déu (Barcelona). ${ }^{h}$ Psicóloga. Centro de Higiene Mental Les Corts. (Barcelona). ${ }^{i}$ Psicólogo. Hospital de Dia Baix Llobregat Parc Sanitari Sant Joan de Déu. Esplugues (Barcelona). ${ }^{j}$ Psicóloga. Servicio de Rehabilitación psicosocial Cornellà Parc Sanitari Sant Joan de Déu (Barcelona). ${ }^{k}$ Psiquiatra. Coordinador de la Unidad de Formación de Parc Sanitari Sant Joan de Déu. Sant Boi de Llobregat (Barcelona). 'Psiquiatra. Directora de Servicios Comunitarios de Parc Sanitari Sant Joan de Déu. Sant Boi de Llobregat (Barcelona). ${ }^{m}$ Psiquiatra. Director de Unidad de Investigación y Desarrollo de Parc Sanitari Sant Joan de Déu. CIBERSAM, Sant Boi Llobregat (Barcelona).

Correspondencia: Susana Ochoa Güerre (sochoa@pssjd.org)

Recibido: 15/10/2010; aceptado: 05/04/2011

\begin{abstract}
RESUMEN: Introducción y objetivos: Pocos son los estudios que evaluan la autopercepción del estigma social en las personas que padecen esquizofrenia. El objetivo del presente estudio consiste en analizar la percepción sobre la esquizofrenia que tienen las personas que la padecen.

Material y métodos: Se realizaron dos sesiones en cuatro grupos focales de personas con esquizofrenia que estaban siendo atendidas en servicios de rehabilitación psicosocial del Parc Sanitari de Sant Joan de Déu. A partir de un guión establecido se valoraron un total de 11 áreas abordadas en los grupos.

Resultados: Las áreas de peligrosidad, culpa, pérdida de roles sociales y miedo al rechazo fueron aquellas que más mencionaron y más preocupaban a las personas que participaron en los grupos focales.

Conclusión: Intervenciones para reducir el estigma social en la comunidad y en los propios usuarios/as deberían ser tenidas en cuenta, especialmente en estas áreas.

PALABRAS CLAVE: estigma social, esquizofrenia, autopercepción, rehabilitación psicosocial.
\end{abstract}

ABSTRACT: Introduction and Objetives: Few studies evaluated the perception of social stigma in people with schizophrenia. The aim of this study was to analyze the perception of people who suffers schizophrenia.

Material and methods: Two sessions in four focus groups of people with schizophrenia who were being treated in psychosocial rehabilitation services Sanitari Parc de Sant Joan de Déu were done. A total of 11 areas (guided by a screenplay) were assessed in the groups.

Results: The areas of danger, guilt, lost of social roles and fear of rejection were those most concerned and most mencioned by the people who took part in focus groups.

Conclusion: Interventions to reduce social stigma in the community and in the self- users should be taken into account, especially in these areas.

KEY WORDS: social stigma, schizophrenia, selfperception, psychosocial rehabilitation. 
ORIGINALES Y REVISIONES

\section{AGRADECIMIENTOS}

Se agradece la colaboración: a las personas que contribuyeron en las sesiones explicando su experiencia sobre su percepción del estigma social; a la beca "Estudios de la Caixa de Sabadell" y al Centro de Investigación en Red en Salud Mental (CIBERSAM) financiado por el Instituto de Salud Carlos III de España.

\section{Introducción}

El estigma es un constructo social que incluye actitudes, sentimientos, creencias y comportamientos (1) que está configurado como prejuicio y conlleva consecuencias discriminatorias hacia la persona estigmatizada.

Las personas que padecen algún trastorno mental son uno de los grupos más estigmatizados de nuestra sociedad, especialmente las que padecen esquizofrenia (2). La enfermedad mental ha estado vinculada a una serie de prejuicios sociales que determinan al enfermo con esquizofrenia como agresivo, raro, impredecible en su conducta, débil, vago e improductivo, con el que no se puede razonar, crónico y culpable de su enfermedad $(3,4)$. Este estereotipo del enfermo mental conlleva una percepción de peligrosidad y un miedo en la relación, que, posiblemente, se ha generado como consecuencia del desconocimiento y la falta de información sobre esta enfermedad (5). Esto tiene como consecuencia que la rehabilitación de las personas con trastornos mentales graves, cuyo objetivo es alcanzar el estatus de ciudadano de pleno derecho, se ve dificultada y en ocasiones impedida (6).

Esta visión de la enfermedad está reforzada por los medios de comunicación, en donde en ocasiones la información es una forma de sesgar la realidad y fomentar un estereotipo de personas agresivas, desorganizadas y que provocan miedo $(7,8)$. Sin embargo, Walsh (2002) (9) demuestra que suelen ser en más ocasiones víctimas de agresiones que la población general, contrariamente al estereotipo social.

En los familiares también están presentes los prejuicios hacia la enfermedad, en donde surgen conductas de vergüenza y de secretismo ligados a un sentimiento de culpabilidad hacia la causa de la enfermedad (10), causando aislamiento (11).

El impacto emocional en las familias tiene una relación directa con esta percepción estigmatizada (12) puesto que el efecto grave del estigma es la internalización de las valoraciones negativas de los otros. Por esto nuestro interés en evaluar el estigma autopercibido, ya que a priori suponemos tendrá consecuencias negativas en el proceso de rehabilitación.

La estigmatización de las personas que padecen un trastorno mental tiene una influencia negativa en cuanto a encontrar un trabajo, una vivienda o de mantener relaciones sociales con amigos o en pareja (13). Por otro lado, estas consecuencias comportan un empeoramiento de su situación llevándoles a una desadaptación social, una baja autoestima, depresión y un aumento de la carga familiar (14). Lo cual 
ORIGINALES Y REVISIONES

dificulta y, a veces, impide una reinserción real en la comunidad, no facilitando el objetivo principal que marca la rehabilitación psicosocial.

Existen pocos estudios que valoren la visión del estigma de quien padece la enfermedad, puesto que existe el prejuicio de que no son capaces de evaluar su propia situación personal debido a al deterioro cognitivo (15). Walh (1999) (16) en un estudio que realizó sobre la visión subjetiva del estigma de trastornos mentales, se encontró que en más del 80\% de los casos habían escuchado comentarios despreciativos sobre la enfermedad mental, habían sido tratados como menos competentes y más de la mitad habían sido esquivados y despreciados.

Ertugrul y Ulug (2004) (17) realizaron un estudio en el que comparaban la presencia o ausencia de estigma social con psicopatología y discapacidad. Los resultados muestran que las personas que tenían una mayor estigmatización de la enfermedad puntuaban más en síntomatología positiva y general, así como en discapacidad.

En Alemania se llevó a cabo un estudio con personas con esquizofrenia que viven en la comunidad, en el que se valoraba la autopercepción del estigma social, a través de grupos focales encuentran cuatro dimensiones en la percepción del estigma: interacción interpersonal, discriminación de estructura, imágenes públicas del enfermo mental y acceso a roles sociales (18). Buizza et al (2005) (19), también con grupos focales encontraron tres dimensiones: distancia física y miedo, distancia social y aislamiento, y responsabilidad social y tolerancia.

La visión subjectiva del que padece la enfermedad en cuanto a cómo es percibido por los otros, especialmente los más próximos, y sobre su enfermedad, influirá directamente en su integración en la comunidad a todos los niveles: vivienda, relaciones sociales, trabajo, etc. Por este motivo, se necesitan hacer estudios para determinar la percepción del estigma social de la persona y así realizar programas de intervención adecuados para dotar al sujeto de recursos y estrategias de afrontamiento ante estas situaciones estigmatizadoras.

Por todo ello, los objetivos planteados en este estudio son analizar la autopercepción del estigma social en personas con esquizofrenia que participan en los programas de rehabilitación psicosocial mediante la información subjetiva de las personas que padecen la enfermedad.

\section{Material y métodos}

\section{Diseño}

Se trata de un estudio cualitativo realizado a partir de la información obtenida en grupos focales con los usuarios. 
ORIGINALES Y REVISIONES

\section{Muestra}

La población a la que nos dirigimos son personas que padecen esquizofrenia, que son atendidas en los servicios comunitarios de rehabilitación psicosocial.

El estudio se realizó en los Servicios de Rehabilitación del Baix Llobregat de la red de salud mental del Parc Sanitari Sant Joan de Déu (Viladecans, Cornellà de Llobregat y El Prat de Llobregat). Estas ciudades están situadas en la periferia de la ciudad de Barcelona y corresponden a áreas ligeramente deprivadas. Cada área cubre una población de entre 90.000 y 130.000 habitantes. Estos servicios están dirigidos a realizar el trabajo de integración del enfermo mental en la comunidad y se atienden principalmente personas con esquizofrenia.

Se informó sobre el estudio a todas las personas que asisten a los Servicios de Rehabilitación del Baix Llobregat y cumplen criterios DSM-IV-R de diagnóstico de esquizofrenia; y se les solicitó su colaboración para participar en el estudio. El total de personas de la población diana fue de 104.

La bibliografía nos indica que la edad, el nivel educativo y el género son factores diferenciales en la valoración de uno mismo, por este motivo se clasificaron al total de usuarios de la muestra en cuatro grupos: menores y mayores de 40 años, nivel educativo primario y medio-alto (estudios secundarios y superiores). Para controlar el género se intentó que los grupos fueran equitativos, incluyendo tanto hombres como mujeres en los cuatro grupos.

De la población diana inicial se realizó una selección aleatoria estratificada por las variables comentadas previamente.

\section{Procedimiento}

El grupo focal es una técnica utilizada en investigación cualitativa que permite poner en contacto las ideas de las personas que participan y que facilita la utilización de un lenguaje inteligible para las personas a las que va dirigido. La técnica consiste en hacer grupos homogéneos y no dirigidos en los que los asistentes puedan conversar sobre el tema indicado dentro de un ámbito informal. A pesar de no ser dirigidos, el coordinador de cada grupo focal contaba con un guión temático para estructurar la sesión y sugerir temas que se considerase importantes para la evaluación del objeto de estudio. Este guión temático fue elaborado y consensuado por los investigadores a partir de la bibliografía sobre el tema y de la propia experiencia, y fue revisado por un experto externo que sugirió ligeros cambios. En la tabla 1 se muestran los contenidos del guión de los grupos focales.

Se realizaron dos sesiones en cada uno de los cuatro subgrupos. A cada subgrupo asistió un terapeuta que guiaba la sesión y un co-terapeuta que se encargaba de anotar las observaciones de las conductas de los miembros del grupo (nerviosismo delante de un tema, silencios...). Los coordinadores de cada grupo fueron psicologos/as o trabajadores sociales de los centros pero se aseguró que no conocieran a 
Tabla 1

Guión temático de los grupos focales

\begin{tabular}{|c|c|c|}
\hline Bloque & Temática & Actividad / Sugerencia preguntas \\
\hline $\begin{array}{l}\text { A) } \\
\text { Introducción }\end{array}$ & $\begin{array}{l}\text { Recepción de participantes } \\
\text { y presentación }\end{array}$ & $\begin{array}{l}\text { Agradecer la presencia de los participantes. Explicación de los objetivos } \\
\text { del estudio, normas de participación }\end{array}$ \\
\hline \multirow[t]{13}{*}{$\begin{array}{l}\text { B) } \\
\text { Temáticas a tratar }\end{array}$} & Pérdida de roles sociales & $\begin{array}{l}\text { ¿Ha pérdido amigos/as por la enfermedad? } \\
\text { ¿Tiene problemas con la pareja? }\end{array}$ \\
\hline & Desresponsabilidad & $\begin{array}{l}\text { ¿Cree que los demás piensan que no tiene capacidad para asumir sus } \\
\text { responsabilidades? }\end{array}$ \\
\hline & Miedo al rechazo & $\begin{array}{l}\text { ¿Cómo ha afectado su enfermedad a las relaciones con los demás?¿cree } \\
\text { que le tratan diferente? ¿le cuesta más relacionarse? }\end{array}$ \\
\hline & $\begin{array}{l}\text { Culpabilidad ligada a la } \\
\text { enfermedad }\end{array}$ & ¿se siente culpable de su enfermedad? \\
\hline & Peligrosidad & ¿Tiene la sensación que los demás temen que sea más agresivo/a? \\
\hline & $\begin{array}{l}\text { Autorrestricción de las } \\
\text { relaciones sociales }\end{array}$ & $\begin{array}{l}\text { ¿Por qué se imagina que la gente piensa que tiene una enfermedad } \\
\text { mental? ¿cómo reaccionó usted y su familia a la enfermedad? }\end{array}$ \\
\hline & Identidad & $\begin{array}{l}\text { ¿Se siente diferente por tener una enfermedad mental en los diferentes } \\
\text { ámbitos de su vida? }\end{array}$ \\
\hline & Pérdida de control & $\begin{array}{l}\text { ¿Cree que ha perdido el control sobre su vida? ¿Depende de otras } \\
\text { personas? }\end{array}$ \\
\hline & Pérdida de capacidades & $\begin{array}{l}\text { ¿Cree que tiene las mismas capacidades? ¿Tiene más problemas de } \\
\text { memoria, atención...? }\end{array}$ \\
\hline & Cronicidad & ¿Cree que su enfermedad puede curarse? \\
\hline & Conciencia de enfermedad & ¿Cree que necesita tratamiento? \\
\hline & Autoritarismo profesionales & $\begin{array}{l}\text { ¿Cree que le tratarían diferente si tuviera otra enfermedad? ¿Se siente } \\
\text { atendido/a? }\end{array}$ \\
\hline & Benevolencia & ¿Le tratan diferente? \\
\hline $\begin{array}{l}\text { C) } \\
\text { Despedida }\end{array}$ & $\begin{array}{l}\text { Despedida y } \\
\text { agradecimiento a los/as } \\
\text { participantes }\end{array}$ & Se les informará de los resultados obtenidos en el estudio \\
\hline
\end{tabular}

las personas que participaban en el grupo focal para facilitar su participación. Los coterapeutas fueron psicologos/as y terapeutas ocupacionales. La duración de las sesiones fue de aproximadamente una hora y media.

Se seleccionaron un total 15 personas en cada uno de los grupos y el terapeuta de referencia del centro de rehabilitación psicosocial informó a los usuarios/as de los objetivos y contenidos del estudio, así como solicitar su colaboración.

Las sesiones de los grupos focales se realizaron fuera del emplazamiento habitual al que asistían los usuarios/as a hacer las actividades. De esta manera se garantizó que las personas que participan en el estudio lo vivieran como externo al 
ORIGINALES Y REVISIONES

servicio de rehabilitación. Por otro lado, dado que las personas que formaban los grupos pertenecen a los diferentes centros de día, posiblemente algunos de ellos no se conocieran entre ellos lo que facilita la vivencia ser externo al servicio.

Las sesiones del grupo focal tienen la intención de facilitar una relación informal por lo que durante la sesión se sirvieron bebidas y aperitivos.

Todas las sesiones de los grupos focales se grabaron y fueron, posteriormente, transcritas.

\section{Análisis de datos}

Se realizó una categorización a partir de los comentarios de los usuarios en las siguientes categorías y temáticas incluidas en el guión de los grupos focales: peligrosidad, culpa ligada a la enfermedad, falta de responsabilidad, pérdida de capacidades, pérdida de roles sociales, miedo al rechazo, cronicidad, pérdida del control de su vida, auto restricción de las relaciones sociales, la idea de que la medicación es también una droga, identidad del enfermo mental, benevolencia. El programa utilizado para el análisis fue el ATLAS-TI.

Aspectos éticos

El estudio fue aprobado por el Comité de Investigación de Sant Joan de Déu y por el Comité de Ética de Sant Joan de Déu.

\section{Resultados}

Los participantes de los grupos focales fueron un total de 15 personas para el primer grupo (menores de 40 años y nivel educativo bajo), 15 para el segundo (mayores de 40 y nivel educativo bajo), 9 para el tercer grupo (menores de 40 años y nivel educativo medio-alto) y 14 para el último grupo (mayores de 40 años y nivel educativo medio-alto).

El total de mujeres de la muestra fue de 15 (28\%), y en el grupo en el que participaron en menor medida fue en el grupo de menores de 40 años con nivel educativo bajo.

Se realizaron grupos de trabajo en los que se analizaron los comentarios registrados durante los diferentes grupos focales. Las categorías previamente diseñadas en los grupos focales en las que los usuarios hicieron más referencia fueron la de peligrosidad, culpa, pérdida de roles sociales y miedo al rechazo. Se han detallado los comentarios relacionados con el estigma social que surgieron en los diferentes grupos para cada una de las categorias comentadas.

En cuanto a peligrosidad (tabla 2) el análisis nos muestra que se identifican diferentes subcategorias. Una de ellas es el miedo hacia las personas que tienen 
ORIGINALES Y REVISIONES

Tabla 2

Comentarios referidos a la categoría de peligrosidad

\begin{tabular}{|l|l|}
\hline Subcategorías & Comentarios \\
\hline Miedo & $\begin{array}{l}\text { - "A mí también cuando a una amiga le dije que había estado en el psiquiátrico me cogió miedo" } \\
\text { - "En plena crisis sí te cogen miedo, porque haces cosas raras, que no es normal y las personas } \\
\text { lo ven y dicen a éste le pasa algo" } \\
\text { - "Siempre les queda el miedo, de qué harás, cómo vendrás, qué sucederá" } \\
\text { - "La gente se asusta de los enfermos mentales y de la enfermedad mental". }\end{array}$ \\
\hline $\begin{array}{l}\text { Relación a otros } \\
\text { colectivos }\end{array}$ & $\begin{array}{l}\text { - "Una persona con un trastorno mental es menos agresiva que una persona con otro trastorno } \\
\text { - "una vez leí que el porcentaje de delitos y acciones violentas es similar al de las personas } \\
\text { "normales" } \\
\text { - "la gente que comete delitos que va borracha o drogada lo ligan con la enfermedad" }\end{array}$ \\
\hline $\begin{array}{l}\text { Pérdida de control } \\
\text { Medicación }\end{array}$ & $\begin{array}{l}\text { - "Pienso que la gente tiene miedo a que pierda el control y sea agresivo y yo también" } \\
\text { - "dicen que uno que tiene esquizofrenia ha matado a alguien pero no dicen que quien está en } \\
\text { tratamiento no mata a nadie" } \\
\text { - "es que sin tratamiento soy más violento y soy más agresivo no puedo estar sin tratamiento". }\end{array}$ \\
\hline $\begin{array}{l}\text { - "Desconocen la enfermedad y por un lado tienen miedo algunos y por otro no saben lo que es". } \\
\text { comunicación }\end{array}$ & $\begin{array}{l}\text { - "miedo a que podamos hacer algo contre ellos, a que tengamos un brote por las informaciones } \\
\text { de la radio, la tele y la prensa de esquizofrenicos que hacen barbaridades a veces". }\end{array}$ \\
\hline
\end{tabular}

una enfermedad mental ("te cogen miedo"). Otra característica que se repite en los grupos es la comparación y la diferenciación entre la peligrosidad de las personas con esquizofrenia y otros colectivos ("alcoholicos, maltratadores...") y con "personas normales", refieriendose a que son más agresivos en otros colectivos y que presentan la misma violencia que las personas sin patologia mental. También surgen temas como que las personas que toman la medicación no son agresivas ("antes eran peligrosos porque no se medicaban"), el desconocimiento de la enfermedad ("desconocen la enfermedad ... tienen miedo algunos...") y la influencia negativa de los medios de comunicación ("...la radio, la tele y la prensa de esquizofrenicos que hacen barbaridades"). Cabe destacar la aparición de comentarios relacionados con la pérdida de control que se recogen en ambas categorías.

La tabla 3 muestra los análisis de la categoría de culpa ligada a la enfermedad. Podemos observar que la culpa está ligada a la causalidad de la enfermedad mental. En diferentes ocasiones en los grupos aparece la idea de que la gente cree que aparece la enfermedad por un exceso de drogas, alcohol o fiestas ("la gente cree que tienes la enfermedad de las borracheras, las drogas"). En relación a este apartado también se comentan los errores que se han hecho en el pasado y la culpabilidad asociada a estos ("te das cuenta de los errores del pasado"). Además aparece ligada a la culpabilidad el sentimiento de el resto de las personas (familia 
Tabla 3

Comentarios referidos a la categoría de culpa ligada a la enfermedad

\begin{tabular}{|l|l|}
\hline Subcategorías & Comentarios \\
\hline Causalidad & $\begin{array}{l}\text { - "Creo que la sociedad piensa que ésta enfermedad viene porque te has pasado con las drogas, } \\
\text { con el alcohol". } \\
\text { - "La gente sólo ve eso, éste se ha ido un montón de fiesta y ha acabado mal" } \\
\text { - "La causa puede ser alguna reacción que te impida evolucionar en la vida con tranquilidad" } \\
\text { - "Dicen que todos los que toman droga acaban en un psiquiátrico" }\end{array}$ \\
\hline Carga & $\begin{array}{l}\text { - "Creo que por tener una enfermedad mental puedo ser una carga para el otro". } \\
\text { - "Me dicen que era un rebelde" } \\
\text { - "antes hacia las cosas mal y te das cuenta de los errores del pasado" }\end{array}$ \\
\hline $\begin{array}{l}\text { Sentimiento de } \\
\text { falsear enfermedad }\end{array}$ & $\begin{array}{l}\text { - "Mi madre piensa que no sirvo para hacer nada, porque como no la ayudo, la culpa la tengo } \\
\text { - "La gente cree que tenemos mucho cuento, que no estamos enfermos" }\end{array}$ \\
\hline
\end{tabular}

y comunidad) creen que tienen "cuento", es decir, que no tienen una enfermedad sino que simulan. El hecho de ser una carga para la familia también aparece como parte de la culpabilidad de tener una enfermedad mental "por tener una enfermedad mental puedo ser una carga para el otro").

En la tabla 4 se recogen algunos comentarios en relación al la pérdida de roles sociales. Se puede observar que la pérdida de roles sociales se basa en pérdida de amistades ("ya no hay amigos como antes"), relaciones sentimentales ("pierdes la oportunidad de ligar") e hijos ("no puedo tener hijos"). También se hace referencia a la pérdida del empleo o de la capacidad de estudio ("no puedo tener un traba-

Tabla 4

Comentarios referidos a la categoría de pérdida de roles sociales

\begin{tabular}{|l|l|}
\hline Subcategorías & Comentarios \\
\hline Amistad & - "Perdí todas las amigas" \\
& - "Ya no hay amigos como antes". \\
& - "Todo eso ya se acabó, el salir y conocer más gente" \\
& - "...no puedo salir por las noches, no puedo beber" \\
\hline Relaciones de & - "No puedo tener hijos" \\
pareja/hijos & - "Estar en tratamiento psiquiátrico crea dificultades para establecer relaciones sentimentales" \\
\hline Trabajo/estudios & $\begin{array}{l}\text { - "No puedo trabajar, no puedo hacer nada, no sirvo para hacer nada, todo me sale mal" } \\
\text { - "Estamos limitados, no trabajas, ni estudias y quedas como un vago/ga" }\end{array}$ \\
& - "No puedes buscar trabajo,primero porque no tienes experiencia y segundo porque estás enfermo, \\
& y a un enfermo nadie lo coge" \\
\hline
\end{tabular}


jo", "no rendia lo suficiente"). Además cabe destacar comentarios de las personas evaluadas en relación a la discriminación sufrida por estos cambios de roles ("no puedes buscar trabajo... porque estás enfermo").

En relación a la categoría de miedo al rechazo aparecen claramente dos aspectos: la discriminación de la sociedad ("huyen de ti", "se cambian de acera") y el ocultamiento que hace la persona de la enfermedad mental para evitar esta discriminación ("yo dije que tenía depresiónporque si digo otra cosa ya cambian y piensan de otra manera") (tabla 5).

En cuanto a la pérdida de capacidades los comentarios generados en los grupos focales están relacionados con la pérdida de la confianza en sí mismo ("he perdido la confianza en mi mismo, no sé si sabré hacer algo...", "no tengo mucha autoestima", "no me valoro"). También hay comentarios que hacen referencia a la visión de los otros sobre esta pérdida de capacidades ("no valoran mis opiniones").

La categoría de desresponsabilidad está muy relacionada con la anterior categoría. Los comentarios surgidos en relación a esta van en la linea de ("hasta que no sea responsable no me iré de casa", "no me dejan cuidar a una persona enferma").

La categoría de identidad del enfermo mental también está presente en otras categorias mencionadas previamente, siendo más amplia de lo contemplado inicialmente. Acciones como eludir mencionar que se tiene una enfermedad mental son las más frecuentemente comentadas ("yo no digo que tengo una enfermedad mental", "cuando me preguntan digo que tengo depresión", "dices que tienes una enfermedad mental y te rechazan", "al decir que eres enfermo mental cambia el trata con los demás"), incluso por parte de la familia ("mi familia no dice que tengo una enfermedad mental").

Tabla 5

Comentarios referidos a la categoría de miedo al rechazo

\begin{tabular}{|l|l|}
\hline Subcategorías & Comentarios \\
\hline Discriminación & - "Cuando sale tu enfermedad las personas huyen, rehuyen de ti" \\
& - "Las amigas se cambiaban de acera y me giraban la cara, perdí todas las amigas". \\
- "Dicen, mira el loco ese" \\
- "Por la calle te señalan" \\
- "Lo que hacen es marginarnos" \\
- "la gente se ríe de nosotros, te dan de lado" \\
- "te juzgan erroneamente por las apariencias"
\end{tabular}


ORIGINALES Y REVISIONES

Los comentarios recogidos en la categoría de autorestricción de las relaciones sociales van en la línea de los comentados previamente en la categoria de pérdida de roles sociales. En esta categoría se recogen más aspectos en relación a la automarginación de los propios pacientes hacia el resto ("he sido yo el que me he apartado de la gente", "intento mantenerme distanciado de las personas", "yo he tenido de marchar de mi barrio porque no me aceptaban").

Las categorías de pérdida de control sobre su vida y benevolencia aportan información similar. Por un lado, las personas entrevistas sienten que han perdido el control sobre su vida y necesitan la supervisión y la sobreprotección de la familia ("me siento supercontrolada por mis padres", "mi madre está siempre detrás de mi", "dependemos un poco", "me siento mimada por mis padres"). En la categoría de benevolencia añaden no solo esta conducta de sobreprotección por parte de la familia sino también por el personal sanitario ("los médicos te tratan con un poco más de cuidado", "estamos demasiado atendidos"). Sin embargo, la postura con respecto al personal sanitario está reflejada en ambos sentidos, por un lado un sentido benevolente (descrito anteriormente) y un sentido autoritario, recogido en la categoría con este nombre ("en los pisos nos controlan demasiado", "no hay libertad", "en los hospitales no deberían atar a la cama").

En relación a la categoria de conciencia de enfermedad surgen dos aspectos importantes: la necesidad del tratamiento y la cronicidad ("me da miedo a dejar la medicación", "se necesita tratamiento para mucho tiempo", la medicación no te cura") y la medicación como equivalente a drogas ("un día iba un poco desequilibrado por la medicación y decian mira ese borracho", "me aburre depender de los medicamentos", "me siento como una rata de laboratorio hasta que aciertan con el medicamento", "la medicación crea dependencia"). Los comentarios relacionados con la categoría de cronicidad de la enfermedad quedan incluidos en las categorías mencionadas anteriormente.

\section{Discusión}

La evaluación del estigma autopercibido por los usuarios/as en procesos de rehabilitación psicosocial mediante la técnica de grupos focales nos ha permitido obtener información sobre las áreas que más preocupan a las personas que padecen esquizofrenia. Las categorías más destacadas, sobre las que los usuarios/as han dedicado la mayor parte del tiempo, en los grupos focales han sido: peligrosidad, culpa, pérdida de roles sociales y miedo al rechazo.

En los estudios previos realizados también a través de grupos focales los resultados obtenidos hablan de cuatro dimensiones (interacción personal, discrimina- 
ORIGINALES Y REVISIONES

ción de estructura, imágenes públicas del enfermo mental y acceso a roles sociales) (18) y de tres dimensiones (distancia física y miedo, distancia social y aislamiento, y responsabilidad social y tolerancia) (19). De alguna manera las dimensiones de roles sociales y distancia social y aislamiento serían similares a la obtenida en nuestro estudio sobre pérdida de roles sociales. El apartado de peligrosidad sería similar al recogido en las dimensiones de distancia física y miedo del estudio de Buizza et al (2005) (19).

La visión de peligrosidad de las personas que padecen una enfermedad mental grave ha sido la más comentada en los grupos siendo uno de los aspectos más trabajados en investigaciones previas Vezzoli et al (2001) (5) sugieren que el miedo hacia el enfermo mental posiblemente viene dado por el desconocimiento de la enfermedad. Como aparece en los grupos el hecho de que los medios de comunicación difundan la imagen de peligrosidad de las personas con esquizofrenia no ayuda a reducir esta connotación sino a potenciar el estereotipo de agresividad del enfermo mental y a generar más miedo en la población general $(7,8)$.

Otro de los aspectos destacados de los grupos es la pérdida de roles sociales lo que hace que hace que esto influya negativamente en aspectos como encontrar un empleo, relaciones sociales y sentimentales. Estos datos coinciden con los factores que destaca Thornicroft et al (2009) (20) en un estudio internacional realizado en 27 paises, ya que los investigadores identifican que en la mayoria de los casos la discriminación hacia las personas con esquizofrenia se da en tres áreas: problemas para hacer amigos, trabajo y una discriminación negativa general. Novak et al (2009) (21) sugiere que el trabajo es uno de los aspectos por los que se discriminan más a las personas con esquizofrenia. Además diferentes estudios comentan que las consecuencias de estos prejuicios negativos y esta discriminación en la búsqueda de empleo y en las relaciones sociales puede comportar un empeoramiento de su adaptación psicosocial (14).

En relación a la culpa y a la causalidad de la enfermedad es importante destacar la visión que las personas con esquizofrenia tienen de lo cree la sociedad sobre ellos. Para las personas que participaron en los grupos era importante diferenciar la figura de la persona que tiene una enfermedad mental de aquella que consume tóxicos porque en la comunidad no se diferencian estos perfiles y se atribuye una relación directa entre consumo de tóxicos y enfermedad mental. Por otro lado, en nuestro estudio aparece ligado este sentimiento de culpabilidad porque la gente cree que son vagos y que no quieren trabajar, esta es una característica de la identidad del propio paciente que también se recoge en estudios previos $(3,4)$.

Es interesante destacar en cuanto a la información relacionada al miedo al rechazo las dimensiones de discriminación de la población general y el comportamiento de ocultamiento de la enfermedad por parte de las personas con esquizofrenia. Estudios previos ya hablaban de la conducta de secretismo por parte de las familias $(10,11)$, pero cabría añadir también este secretismo por parte de los 
ORIGINALES Y REVISIONES

propios usuarios/as.

Por último se deberían tener en cuenta también los aspectos relacionados con la pérdida de capacidades que influeyen en la autoestima directamente, la pérdida de control de su propia vida y la influencia de la familia y profesionales en el trato hacia las personas que sufren esquizofrenia.

El hecho de identificar qué aspectos son los que preocupan más a las personas con esquizofrenia y facilitan que sean discriminados resulta de utilidad para implementar estrategias que favorezcan la reducción del estigma social en la comunidad y en las propias personas que lo padecen.

BIBLIOGRAFÍA:

(1) López-Ibor, JJ., Cuenca, O. La esquizofrenia abre las puertas. Programa de la Asociación Mundial de Psiquiatría para combatir el estigma y la discriminación debidos a la esquizofrenia. Madrid: Lilly, 2000.

(2) Domenici, PV. Mental health care policy in the 1990s: discrimination in health care coverage of seriously mental ill. J Clin Psychiatry, 1993, 54, 5-6.

(3) Byrne, P. Psychiatric stigma. Br J Psychiatry, 2001, 178, 281-284.

(4) Crisp, AH., Gelder, MG., Rix, S., Meltzer, MI., Rowlands, OJ. Stigmatisation of people with mental illnesses. Br J Psychiatry, 2000, 177, 4-7.

(5) Vezzoli, R., Archiati, L., Buizza, C., Pasqualetti, P., Rossi, G., Pioli, R. Attitude towards psychiatric patients: a pilot study in northern Italian town. Eur Psychiatry, 2001, 16 (8), 451-458.

(6) Rodríguez, A. Rehabilitación psicosocial de las personas con trastornos mentales crónicos. Madrid: Pirámide, 1997.

(7) Ferriman, A. (2000). The stigma of schizoprhenia. Br J Med, 24, 281-283

(8) Boisvert, CM,, Faust, D. Effects of the label schizophrenia on causal attributions of violence. Schizophr Bull, 1999, 25 (3), 479-491.

(9) Walsh, E., Buchanan, A., Fahy, T. Violence and schizophrenia: examining the evidence. Br J Psychiatry, 2002, 180, 490-5

(10) Vaughn, CE., Leff, JP. The influence of family and social factors on the course of psychiatric illness. Br J Psychiatry, 1976, 129, 125-137.

(11) Magliano, L., Marasco, C., Fiorillo, A., Malangone, C., Guarneri, M., Maj, M., Working Group of the Italian National Study on Families of Persons with Schizophrenia. The impact of professional and social network support on the burden of families of patients with schizophrenia in Italy. Acta Psychiatr Scand, 2002, 106 (4), 291-298.

(12) Phillips, M., Pearson, V., Li, F., Xu, M., Yang, L. Stigma and expressed emotion: a study of people with schizophrenia and their family members in China. Br J Psychiatry, 2002, 181, 488-93.

(13) Penn, D., Martin, J. The stigma of severe mental illness: some potencial solutions for a recalcitrant problem. Psychiatr Q, 1998, 69, 235-247.

(14) Autonell, J., Ballús-Creus, C., Busquets, E. Estigma de la esquizofrenia: factores implicados en su producción y métodos de intervención. Aula Médica de Psiquiatría, 2001, 1, 53-58.

(15) Thompson, AH., Stuart, H., Bland, RC., Arboleda-Florez, J., Warner, R., Dickson, RA., Sar- 
torius, N., Lopez-Ibor, JJ., Stefanis, CN., Wig, NN., WPA. World Psychiatric Association. Attitudes about schizophrenia from the pilot site of the WPA worldwide campaign against the stigma of schizoprhenia. Soc Psychiatry Psychiatr Epidemiol, 2002, 37 (10), 475-482.

(16) Wahl, O. Mental Health Consumers' Experience of Stigma. Schizophr Bull, 1999, 25 (3), $467-478$

(17) Ertugul, A., Ulug, B. Perception of stigma among patients with schizophrenia. Soc Psychiatry Psychiatr Epidemiol, 2004, 39, 73-77.

(18) Schulze, B., Angermeyer, MC. Subjective experiences of stigma. A focus group study of schizophrenic patients, their relatives and mental health professionals. Soc Sci Med, 2003, 56 (2), 299312.

(19) Buizza, C., Bertocchi, E., Rossi, G., Pioli, R. La percezione dello stigma del punto di vista delle persone che soffrono di schizophrenia. Psichiatria di Comunità, 2005, 4 (1), 37-45.

(20) Thornicroft, G., Brohan, E., Rose, D., Sartorius, N., Leese, M. INDIGO Study Group. Global pattern of experienced and anticipated discrimination against people with schizophrenia: a cross-sectional survey. Lancet, 2009, 31, 373(9661), 408-15.

(21) Novak, L., Svab, V. Antipsychotics side effects' influence on stigma of mental illness: focus group study results. Psychiatr Danub, 2009, 21(1), 99-102. 